\title{
DISPERSION ANALYSIS OF MICROSTRIP LINES WITH NONRECTANGULAR CROSS-SECTIONS
}

\author{
'Jean-Fu Kiang, ? Chung-I G. Hsu, ,Ching-Her Lee \\ 1 \\ Graduate Institute of Communication Engineering \\ National Taiwan University \\ Taipei, Taiwan 10617, ROC \\ "Department of Electrical Engineering \\ Da-Yeh University \\ Changhua, Taiwan 515, ROC \\ ${ }^{3}$ Department of Industrial Education \\ National Changhua University of Education. \\ Changhua, Taiwan 500, ROC
}

\begin{abstract}
A volume integrai equation formulation incorporated with the Galerkin's method of moments is proposed to compute the conductor loss of multilayered microstrip lines The effects of the conductor thickness, the strip crosssectional shape. and the proximity of the gromd planes on the dispersion relation are examined. Results show that. due to skin effect. the conductor loss is larger for the case with longer strip cross-sectional contour. It is also found that the conductor loss is less sensitive to the strip thickness as the later is larger than three skin depths. Moving the ground planes closer to the conductor strip will result in latger loss, as was obseried in this work.
\end{abstract}

\section{INTRODUCTION}

In high-density. high-speed microwave integrated circuits, the conductor and dielectric losses in microstriptype interconnects and line stnuctures are of particular certain. As the operating frequency is getting ligher, they should be taken into account to accurately predict the electronagnetic belaviors of the circuits. Conventionally, to compute the conductor loss. a perurbation approach has usually been used [1]. The conductor loss is treated as a pertinrbation in the lossless case, and is evaluated by using the surface resistance and the surface current. Other approaches, such as the integral equation method that is derived using the equivalent sterface impodance in the boundary condition [2]. or as the approximate method proposed by Whecler [3] that is based on the incremental inducunce mile, are also used to calculate the conductor loss. All these tricthods assuned that the strip thickness is larger than the skin depth. In practical application. however, the advanced fabrication process may result in conductors that are very thin, so that conventional perturbation method could lose irs accuracy in computing the dissipation of the microstrip lines. In [4]. Kiang proposed an volume integral equation formulation using the dyadic Green's function to solve for the dispersion relation of the single and the coupled conductor strips. The microstrips considered in this technique can be very thin and their cross sections can be of arbitrary shape.

In this work. a volume integral equation formulation is employed to analyze the multilayered microstrip lines. The dyadic Green's function for the planar layered media is derived from a multi-sectioned transmission-line network analog [5]. In the formulation, the number of the background dielectric layers is assumed arbitany, and the dielectrics on be lossy. In addition the microstrip lines of arbitrary crosssection can reside in different layors. For numerical solution. the method of moments is implemented To do that, the cross-section of the conductor strip is first divided into small cells. Then, the eigenmode clectric fjelds on the cross section are represented by a set of pulse basis functions. Following Galerkints procedure, the same set of basis functions is chosen to test the integral equation. The montent method procedure will result in a mitrix equation, from which both the phase and attenuation constant are solved. In this research, the multilavered structures with conductor thickness comparable to tho skin depth are of primary interest. The dispersion characteristics of conductor strips having recrangular, trapezoidal, and fish-eyed cross-sections are studied. The effects of the conductor thickness and the proximity of the ground planes on the dispersion relation are also examined

\section{T'HEORY AND ANALYSIS}

Consider the configuration of the multilayered structure with conducting strips embedded in layers $\ell$ and $m$, as depicted in Fig. I. Botl the diclectrics and the conductors are assumed to be lossy in nature. To solve the problem, the spectral Green's function for the plastar mutitilayered 
medium is first developed. Then, assuming uniformity along the propagation direction $y$ for the whole structure, and an $e^{j / 2 t}$ time dependence, the electric fields in layer $\ell$ can be expressed, in terms of the dyadic Green's function, as

$$
\vec{E}(\vec{r})=\int_{V_{1}} d \nu^{\prime} \vec{G}\left(\vec{r} ! \vec{r}^{\prime}\right) \cdot \vec{J}_{e q}\left(\vec{r}^{\prime}\right)
$$

or

$$
\begin{aligned}
& \bar{E}(\vec{r})=\int_{r_{t}} d N^{r}\left[\tilde{G}^{P V}\left(\vec{r} \mid \vec{r}^{\prime}\right)+\frac{j}{\omega \varepsilon_{0} \varepsilon_{r t}^{\prime}} \delta\left(\ddot{r}-\vec{r}^{r}\right) \hat{z} \bar{z}\right] \cdot \vec{J}_{s q}\left(\vec{r}^{\prime}\right) \\
& =\hat{z} \frac{j}{\alpha \varepsilon_{1)} \varepsilon_{r l}} \Delta \sigma(\vec{r}) \bar{E}_{s}(\vec{r}\rangle+\int_{\vec{F}_{s}} d \nu^{\prime} \vec{G}^{P V}\left(\vec{r} \mid \vec{r}^{\prime}\right) \cdot \bar{J}_{\mathrm{eq}}\left(\vec{r}^{\prime}\right)
\end{aligned}
$$

where $\varepsilon_{r t}=\varepsilon_{r t}^{r}-j\left(\sigma_{t} / \omega \varepsilon_{0}\right) \cdot$ is the complex-valued relative permittivity of tayer $\ell, \vec{G}^{P V}\left(\vec{r} \mid \vec{r}^{\prime}\right)$ is the principal-value part of the dyadic Green's function, and $\frac{j}{\omega \varepsilon_{0} \varepsilon_{r \ell}^{\prime}} \delta\left(\vec{r}-\bar{r}^{\prime}\right) \hat{z} \hat{z}$ is the source dyadic. Equation (2) is the needed volume integral equation, in which $V_{\ell}$ is the volume occupied by the conductors. Define the Fourier transform pair as

$$
\vec{G}^{P Y}\left(\vec{r} \mid \vec{r}^{\prime}\right)=\frac{1}{4 \pi^{2}} \iint_{-\infty}^{n} d \vec{k}_{p} e^{-\left\langle\vec{k}_{p}(\vec{p}-\vec{p})\right.} \ddot{G}^{P V}\left(\vec{k}_{p} ; z \mid z^{\prime}\right)
$$

where $\bar{r}=\vec{\rho}+\hat{z} z, \quad \bar{\rho}=\hat{x} x+\hat{y} y$, and $\quad \bar{k}_{p}=\hat{x} k_{x}+\hat{y} k_{y}$ Then (2) can be rewritten as

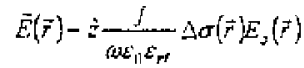

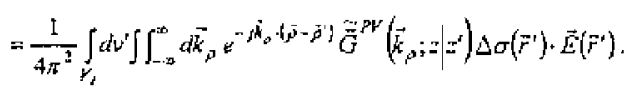

For a wave propagating along the axial direction of the conducting strips, the electric field can be expressed as

$$
\bar{E}(\vec{r})=\bar{E}\left(\vec{r}_{s} k^{-j \pi \nu},\right.
$$

where $\bar{r}_{s}=\hat{x} x+\hat{z} z$, and $\eta$ is the propagation constant of the eigenmode that is yet to be determined. Thus, (4) becomes

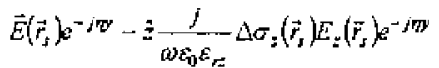

$$
\begin{aligned}
& =\frac{1}{4 \pi^{2}} \int_{s_{t}} d d s^{\prime}\left\{\int_{-\infty}^{n} d b^{\prime} \int_{-\infty}^{n} d k_{y} e^{-j k_{1}\left(y-y^{\prime}\right)} \int_{-\infty}^{m} d k_{x} e^{-j k_{1}\left(x+x^{2}\right)}\right. \\
& \left.\vec{\sigma}^{p v}\left(k_{x}, k_{y} ;=\mid z^{\prime}\right) \Delta \sigma\left(\vec{F}_{s}^{\prime}\right) \cdot \vec{E}\left(\vec{r}_{s}^{\prime}\right) e^{-j \sigma^{\prime}}\right\} \text {. }
\end{aligned}
$$

where $S$, is the cross-sectional area of the conductor strips in layer $\ell$. Note that

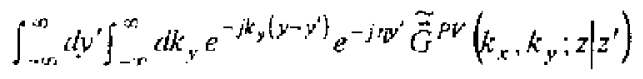

$$
\begin{aligned}
& =2 \pi \int_{u \rightarrow \infty}^{n} d k_{y} e^{-j k, y} \mathscr{G}^{P} P\left(k_{x}, k_{y}: z \mid z^{\prime}\right) b\left(k_{y}-\eta\right) \\
& =2 \pi e^{-\lambda W^{\prime}} \tilde{G}^{F}\left(k_{x}, m ; z_{1} z^{\prime}\right) \text {. }
\end{aligned}
$$

Hence, by using (7) in (6) and dropping the factor $e^{-j r y}$ we obtain

$$
\begin{aligned}
& \left.E\left(\ddot{r}_{s}\right)-\dot{z} \frac{j}{\omega \varepsilon_{0} \varepsilon_{r}}\right\lrcorner \sigma\left(\ddot{r}_{s}\right) E_{z}\left(\bar{r}_{r}\right)
\end{aligned}
$$

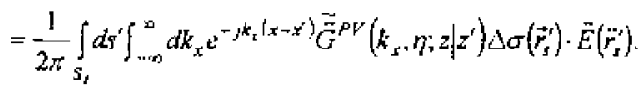

Equation (8) is the final form of the integral equation for the multilayered structure with conductor strips in only one layer. For the case of multiconductors in different layers, the integral equation is similar to that of (1) (or (8)), and can be derived accordingly,

To obtain numerical solution, the integral equation (8) is solved using Gralerkin's method of moments. The eigenmode electric field on the conductor cross-section $S$ is represented by a set of pulse basis functions as

$$
\vec{E}(\vec{p})=\sum_{n=1}^{W}\left(\hat{x} a_{n x}+\hat{y} a_{n y}+\hat{z} a_{n z}\right) P\left(\frac{x-x_{n}}{\Delta x_{n}}\right) P\left(\frac{z-\bar{z}_{n}}{\bar{y}_{n}}\right)
$$

where

$$
P(t)= \begin{cases}1, & |f| \leq 1 / 2 \\ 0, & \text { otherwise }\end{cases}
$$

and $\Delta x_{n}$ is the length of the nth segment in the $x$ direction. $\Delta \bar{z}_{n}$ is that of the $n$th segment in the $z$ direction, with $1 \leq n, m \leq Y$. The center coordinate of the $(n, m)$ cell is denoted as $\left(x_{n_{r}} \bar{z}_{m}\right)$. Then the same sel of basis functions is chosen as the testing functions to construct a matrix equation. from which both the phase constant and the attenuation constant are solved.

In this study, the multilayered microstrip structures with conductor thickness on the order of the skin depth arc of primary interest. The approach introduced above will be used to analyze such structures. Computed results and the pertaining discussions are presented in the following scction.

\section{RESULTS AND DISCUSSION}

In this section, the computed tesults of some typical sample stnictures of Fig. I are presented. To validate the ontlined approach, we first show, in Fig. 2, the attenuation constant of a microstrip line of width 258 versus the thickness to skin depth ratio, $v \delta$. The results from [6] are also plotted for comparison, and good agreement was observed. Next, wo examine the effect of cross-sectional shape on conductor loss. In Fig. 3, we present the dispersion relation of the attenuation constant of a stripline for three different conductor cross-sections. With the area of the cross-section 
fixed. the fisl-eyed case. which has relatively larger crosssectional contour. has higher conductor loss. This belavior is seen to be more prenouliced in high frequency region due to skifi effect. In addition to the cross-sectional shape effects, wic are also interested to know the dependence of the dispersion betavitor on the dielectric constants of the background media. In Fig. + t. we plot the attenuation constant of a stripline embedded in the interface of two dielectic layers. With $\varepsilon_{1}$ fixed we see that larger $\varepsilon_{2}$ will cause higher conductor loss (see Fig + (a)). This is due to the fact that in this case the field between the strip line and the upper shielding conducting plane is stronger, and hence will also be the case for the field inside the conductor region near the upper strip surface. It is aiso seen in Fig 4 (b) that increasing $\varepsilon_{2}$ will give larger phase constant. Finally, we present in Fig. 5 the effecls of strip thickness and the proximity of the ground planes on conductor loss. Results revenl that when the strip thickness is increased bevond three skin depths. the conductor ioss is approaching a constant value. Also, the loss will increase if the ground planes are moved closer to the conducting strips.

\section{CONCLUSIONS}

In this work, the multilayered microstrip lines with conductor thickness comparable to the skin depth are sludied using a volume integral equation formulation incorporated with the Galerkin's method of moments. Results show that, due to the skin effect, the conductor loss is larger for the case with longer strip cross-sectional contour. The conductor loss is less sensitive to the strip thickness as the later is larger than three skin dopths. The study of the effects on the conductor loss due to relative permittivity of the background medium reveals that higher dielectric constant will cause larger toss. Also. decreasing the separation between the ground planes and the conductor strip will increase the conductor loss.

\section{ACKNOWLEDGEMENT}

This work was supported by the Industrial Technology Research Institute (ITRI), Taitwan, R O. C., under contact G.t- 88007-A.

\section{REFERENCES}

[1] R. F. Harrington and C. Wci, "Losses on пnuliconductor transmissin lines in a multilayered dielectric nedia." IEEE Trans. Microwave Theory Tccl.. vol. MTT-32. pp. 705-710. Juiy 1984.

[2] T. E. Van Deventer. P. B. Katchi, and A. C. Cangellaris. "An integal equation method for the cvaluation of conductor and dielectric losses in highfrequency interconnects." IVEE Trans. Microwave Thieory Tech, vol 37. pp. 196+-1972, Dec. 1989.

[3] H. A. Whecler. "Formulas for the skin effect." Proc. IRE, vol 30. pp. $\$ 12-42+$ Sep. $19+2$

[4] J. F. Kang. "Integral equation solution to the skin cffect problem in conductor strips of finite thickness," [EEE Trans. Microwate Theory Tech.. vol. 39, no. 3, pp. 452-460, Mar. 1991.

[5] K A Michalski, and J.R Mosig, "Multilayered media Green's functions in integral equation formulations." IEEE Trants. Antennas Propagat, vol, 45, no. 3, pp. 508-519. Mar. 1997.

[G] L. P. Vakanas, A. C. Cangellaris, and J. L. Prince, "A parametric study of the attenuation constant of lossy mictostrip lines," IEEE, Trans, Microwave Thcory. Tech.. vol. 38, no. 8. pp. 1136-1139, Aug. 1990.

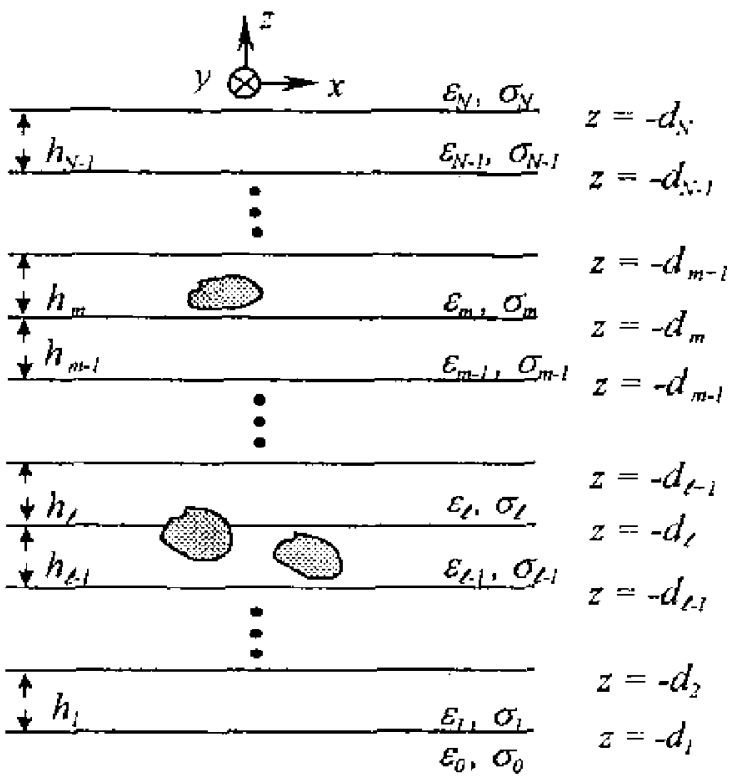

Fig. I Conductors of arbitrary cross section embedded in layered medium.

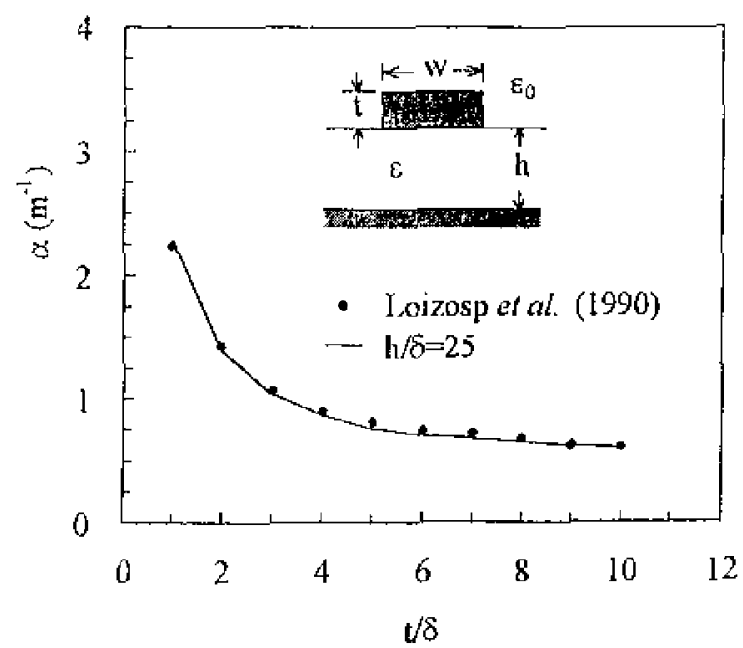

Fig. 2 Attenuation constant for a tnictostrip of width 258 ploted versus the thickness to skin depth ratio, $t / \delta .(f=1$ $\mathrm{GH}$. $w=62.5 \mu \mathrm{In}, \delta=2.5 \mu \mathrm{m}, z=11 \varepsilon_{0,}, \sigma=4 \times 10^{7} \mathrm{~S} / \mathrm{m}$. loss tangent $=2.5 \times 10^{-4}$ ) 


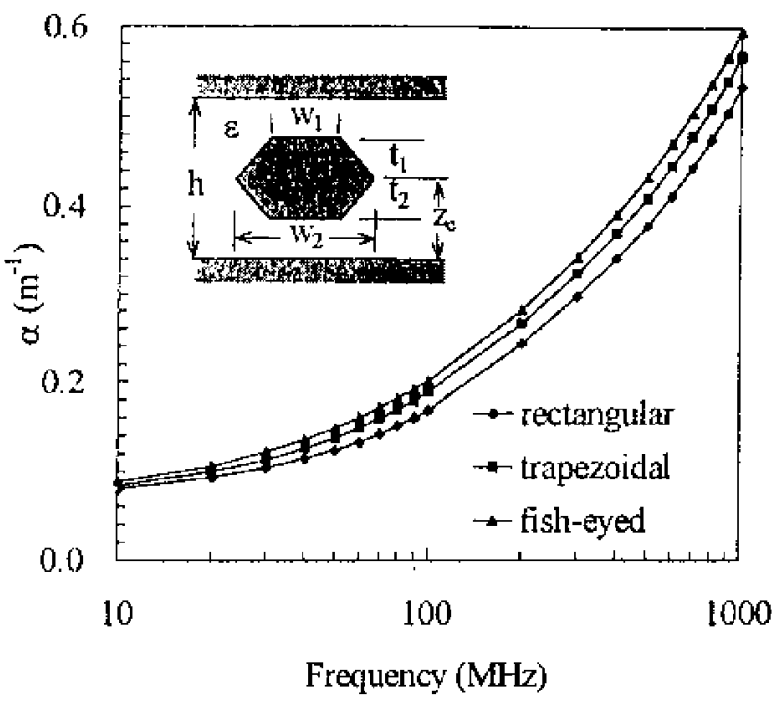

Fig. 3 Dispersion relation of striplincs with the same cross-5ectional area but different shapes. For rectangular case, $w_{1}=w_{2}=100 \mu m, t_{1}=t_{2}=12.5 \mu m$, for trapezoidal case, $w_{1}=75 \mu \mathrm{m}, w_{2}=125 \mu \mathrm{m}, t_{1}=25 \mu \mathrm{m}, t_{2}=0 \mu \mathrm{m}$, and for fish-eyed case, $w_{1}=75 \mu \mathrm{m}, w_{2}=125 \mu \mathrm{m}, t_{\mathrm{l}}=\mathrm{t}_{2}$ $=12.5 \mu \mathrm{m}$. Also $\varepsilon=10 \varepsilon_{0}, \sigma=5.92 \times 10^{7} \mathrm{~S} / \mathrm{m}, \mathrm{h}=600$ $\mu \mathrm{m}, \mathrm{z}_{\mathrm{q}}=300 \mu \mathrm{m}$.

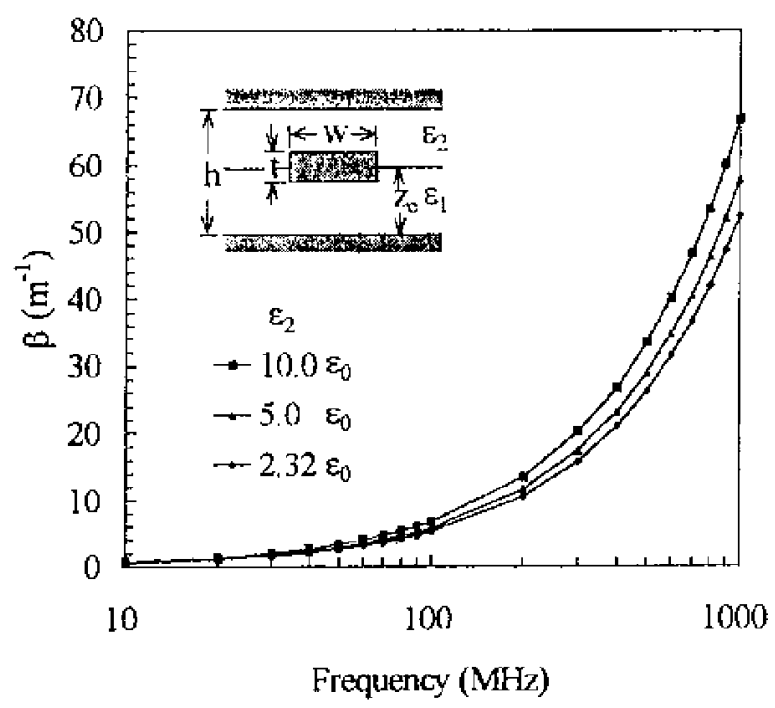

(a) Phase constant

Fig. 4 Dispersion relation of a stripline embedded in the interface of two layers: $w=100 \mu \mathrm{m}, \mathrm{t}=25 \mathrm{\mu m}, \mathrm{h}$ $=600 \mu \mathrm{m} . z_{\mathrm{u}}=\mathrm{h} / 2, \varepsilon_{\mathrm{l}}=10 \varepsilon_{0}, \sigma=5.92 \times 10^{7} \mathrm{~S} / \mathrm{tn}$.

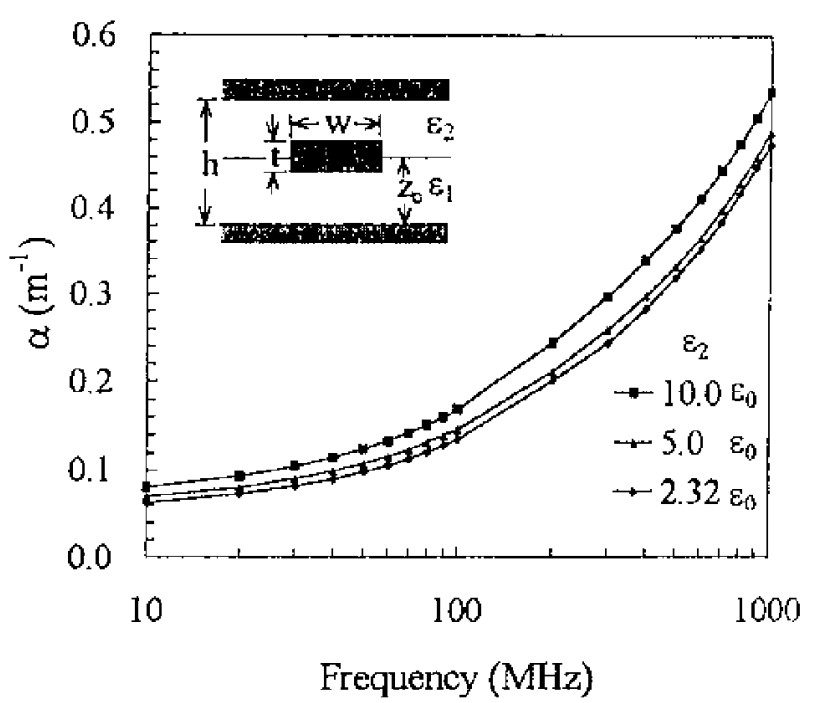

(b) Attenuation constant

Fig. 4 (continued)

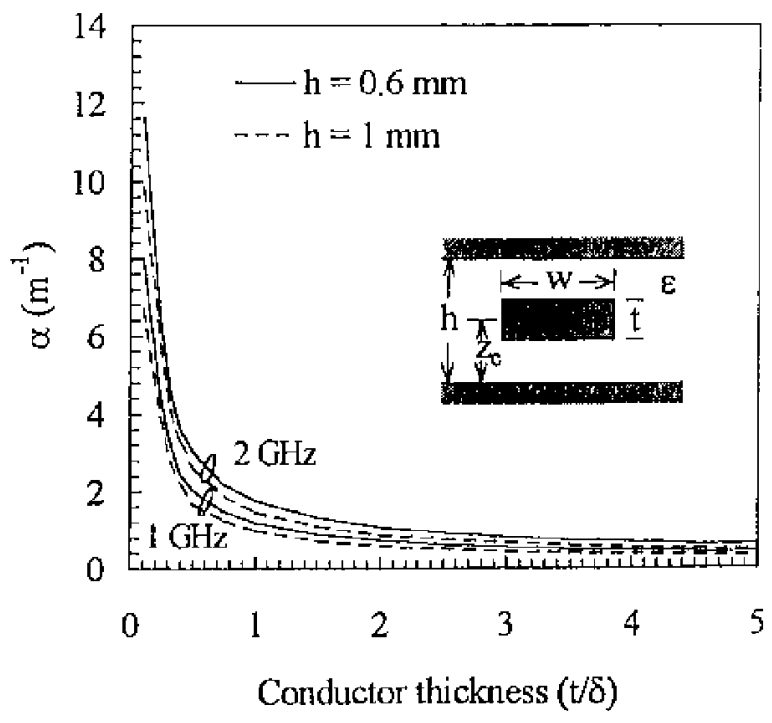

Fig.5 Attenuation constants with diffferent str.p thickness and proximity of the ground planes, $w$ $=100 \mu \mathrm{m}, \sigma=5.92 \times 10^{7} \mathrm{~S} / \mathrm{m}, \mathrm{s}_{\mathrm{t}}=10 \varepsilon_{\mathrm{p}}, \mathrm{z}_{\mathrm{c}}=\mathrm{l} \mathrm{V} / 2$. 